\title{
Fluid Overload in Critically III Patients with Acute Kidney Injury
}

\author{
Jorge Cerda ${ }^{a, b}$ Geoffrey Sheinfeld ${ }^{c}$ Claudio Ronco ${ }^{d, e}$ \\ ${ }^{a}$ Albany Medical College, and ${ }^{b}$ Capital District Renal Physicians, Albany, New York, N.Y., and ${ }^{\circ}$ Department of \\ Surgical Critical Care R. Adams Cowley Shock Trauma Center, University of Maryland Medical Center,

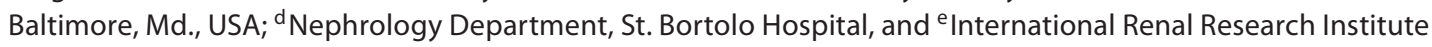 \\ of Vicenza (IRRIV), Vicenza, Italy
}

\section{Key Words}

Fluid overload · Sepsis · Acute kidney injury • Renal

replacement therapy

\begin{abstract}
Fluid overload may occur in critically ill patients as a result of aggressive resuscitation therapies. In such circumstances, persistent fluid overload must be avoided since it does not benefit the patient while it may be harmful. In the septic patient, early volume expansion seems to be beneficial. Beyond that threshold, when organ failure develops, fluid overload has been shown to be associated with worse outcomes in multiple disparate studies. One well-designed randomized controlled trial showed the benefit of a conservative fluid management strategy based on limited fluid intake and use of furosemide in such patients. Use of diuretics should be only short term as long as it is effective, generally at high doses, while avoiding simultaneous utilization of nephrotoxins such as aminoglycosides. Multiple randomized controlled trials have not shown benefit in the use of diuretics, either to prevent AKI or to treat established AKI. If fluid overload (defined as fluid accumulation $>10 \%$ over baseline) develops and the patient does not respond to diuretics, persistent use of these drugs will only lead to a delay in the initia-
\end{abstract}

tion of dialysis or ultrafiltration and an increased risk of negative patient outcomes. In that setting, early initiation of continuous renal replacement therapies may be preferable.

Copyright $\odot 2010$ S. Karger AG, Basel

\section{Introduction}

The incidence of acute kidney injury (AKI) among critically ill patients is as high as $20 \%[1,2]$; diagnosis of this condition is difficult and, often, late. Devoid of effective therapeutic ways to restore renal function, clinicians are limited to ensure optimization of fluid volume and composition [3], and to provide hemodynamic, nutritional support and renal replacement therapy (RRT) when indicated [4] while awaiting spontaneous renal recovery.

Oliguric AKI, commonly attributed to a more severe degree of renal injury, is associated with worse survival than the nonoliguric form [5]. In an attempt to control fluid overload, managing clinicians often endeavor to restore diuresis, expecting that the conversion from oliguric to nonoliguric renal dysfunction will improve patient outcome. In reality, response to diuretics only indicates lesser severity of disease; diuretics do not improve func-

\section{KARGER \\ Fax +41613061234 E-Mail karger@karger.ch} www.karger.com
(C) 2010 S. Karger AG, Basel 0253-5068/10/0294-0331\$26.00/0

Accessible online at: www.karger.com/bpu
Jorge Cerda, MD

Capital District Renal Physicians

62 Hacket Blvd

Albany, NY 12209 (USA)

Tel. +1 518434 2244, Fax +1 518434 4659, E-Mail cerda@ nycap.rr.com 
tional outcomes [6] and may be associated with worse patient and functional survival [7-9]. Their use should be limited to optimizing fluid management whenever possible, and to help control electrolyte disorders including hyperkalemia, hypermagnesemia and hypercalcemia [7]. Given the importance of prompt correction of fluid overload, when diuretics fail renal replacement therapies should be initiated without delay.

Among critically ill patients with AKI, several observational studies initially performed in the pediatric population [10-13] suggest an association between fluid overload and increased mortality. An adverse effect of fluid overload on outcomes has been reported in other critically ill patients without AKI, including patients with septic shock and acute lung injury [14-19]. In these latter patient groups, aggressive fluid resuscitation is frequently provided to manage hypotension [20]. Unfortunately, this approach often results in significant fluid overload, especially when renal function is compromised [21].

On this subject, a 'chicken and egg' debate has occurred, one side arguing that severe fluid overload indicates a very sick patient who requires large volumes of fluid to maintain hemodynamic stability in the context of pronounced critical illness. On the other side, increasing evidence shows that fluid overload itself could be seen as a 'biomarker' with intrinsic toxic effects on organ function, contributing independently to the elevated mortality of critically ill patients $[8,22]$.

In this article, we will successively discuss the evidence showing the association between fluid overload and patient outcomes among hemodynamically unstable patients with and without AKI, and briefly review current knowledge on techniques available for volume control.

\section{Fluid Balance in Critically III Patients}

\section{Critically Ill Patients without AKI}

In a landmark randomized controlled trial (RCT) performed by Rivers et al. [23], a highly protocolized resuscitative approach (early goal-directed therapy; EGDT) was compared to conventional management in 263 emergency department patients admitted with sepsis-induced hemodynamic compromise. This treatment bundle formed the basis of the Surviving Sepsis Campaign guidelines [24]. Confirmatory EGDT studies are currently being carried out in a larger, multicentric 5-year RCT of alternative resuscitation strategies for septic shock (the ProCESS trial, ClinicalTrials.gov Identifier
NCT00510835). This study is currently assessing the reproducibility of the initial findings. In the meantime, variations of this strategy are widely applied around the world [25].

The EGDT, conducted for $6 \mathrm{~h}$ in the emergency department, involved adjustments of cardiac preload, afterload, and contractility to balance oxygen delivery with oxygen demand. Central venous pressure, mean arterial pressure, and central venous oxygen saturation were corrected to predetermined targets. A major aspect of the protocol included initially aggressive volume administration. While the total amount of volume administered within the first $72 \mathrm{~h}$ was approximately the same in both arms (a mean of 13,357 \pm 7,729 vs. 13,443 $\pm 6,390 \mathrm{ml}$ ), patients randomized to EGDT received the majority of volume within the first $6 \mathrm{~h}$, and patients on standard therapy received the infusion between 7 and $72 \mathrm{~h}$ after admission. Rather than the amount of volume administered, the critical difference was the timing of the administration: early up-front aggressive resuscitation was effective, but late and excessive fluid administration was associated with worse survival at hospital discharge, 30 and 60 days. The survival difference was significant in patients with septic shock and severe sepsis but not in those with sepsis syndrome.

The mean serum creatinine in both groups was 2.6 $\mathrm{mg} / \mathrm{dl}$ at baseline, suggesting a high incidence of AKI even before fluid administration began, although inclusion of end-stage renal disease patients could partly explain the elevated mean serum creatinine at baseline. A significant percentage (more than $20 \%$ ) of patients had pre-existing renal insufficiency, placing them at increased risk for the development of AKI.

Rivers et al. [23] did not specifically report the percentage of patients with AKI at entry and the subsequent development of AKI (including the need for renal replacement therapy) after treatment interventions. As severe sepsis and septic shock have been shown to be risk factors for AKI $[26,27]$ and in view of the relatively high mean baseline serum creatinine, a reasonable assumption is that AKI progressed in a significant percentage, with many eventually requiring renal replacement therapy. Based on the volume of fluid used for resuscitation, the possibility that many patients, especially in the conventional management group, needed dialysis driven by AKI and fluid overload is concerning. The clinical implications of fluid overload in sepsis syndromes (see below) raise questions about the EGDT study.

Alsous et al. [14] retrospectively evaluated the effect of fluid overload on outcome in septic shock in 36 patients. 
Mortality outcomes were adjusted for age, baseline APACHE II score, SOFA score on days 1 and 3, and the need for mechanical ventilation. For patients in whom a net negative fluid balance of at least $500 \mathrm{ml}$ could be achieved within the first 3 days of ICU hospitalization, survival was $100 \%$. Conversely, survival of patients in whom net negative fluid balance could not be attained was only $20 \%$. During the 7 -day analysis period, daily fluid balance on average was always more positive among patients who died than those who survived. From day 2 to day 7 , surviving patients essentially attained neutral fluid balance on a daily basis, while nonsurviving patients had mean positive fluid balance ranging from 1 to 4 liters per day. The differences between surviving and nonsurviving patients were greatest (approximately 3.54 liters) on days 2 and 3.

Combining aspects of both the Rivers and Alsous [14, 23] studies, Murphy et al. [15] recently performed an observational analysis of 212 patients who developed acute lung injury in the setting of septic shock. These investigators assessed the effects of timing of fluid resuscitation and control of fluid balance on patient outcome. Adequate initial fluid resuscitation was achieved by administration of an initial fluid bolus of $\geq 20 \mathrm{ml} / \mathrm{kg}$ prior to and a central venous pressure of $\geq 8 \mathrm{~mm} \mathrm{Hg}$ within $6 \mathrm{~h}$ after the onset of vasopressor therapy. Conservative late fluid management was defined as neutral or negative fluid balance on at least 2 consecutive days during the first 7 days after the onset of septic shock.

Patients meeting both the initial resuscitation and fluid balance criteria showed the lowest hospital mortality, while patients meeting only one or neither of these criteria had significantly worse outcomes. Hospital mortality in patients meeting both criteria was $18.3 \%$, compared with $77.1 \%$ among those meeting neither $(p<0.001)$ (fig. 1). Consistent with the Alsous study, daily fluid balance in nonsurviving patients was significantly greater (and always positive: $+2,500$ to $+1,000 \mathrm{ml}$ ) than in surviving patients $(+1,300$ to $-500 \mathrm{ml})$ on days $3-7$, while surviving patients achieved zero daily fluid balance on day 4. Moreover, cumulative fluid balance differed significantly between nonsurviving and surviving patients during the same time period (day 7 values +13.7 and +8.0 liters, respectively). Multivariate regression analysis demonstrated that the inability to achieve conservative late fluid management (a likely surrogate for fluid overload) was an independent mortality risk factor (adjusted OR $6.13 ; \mathrm{p}<0.001$ ).

In a multicentric observational cohort including 198 ICUs from 24 European countries (the Sepsis Occurrence

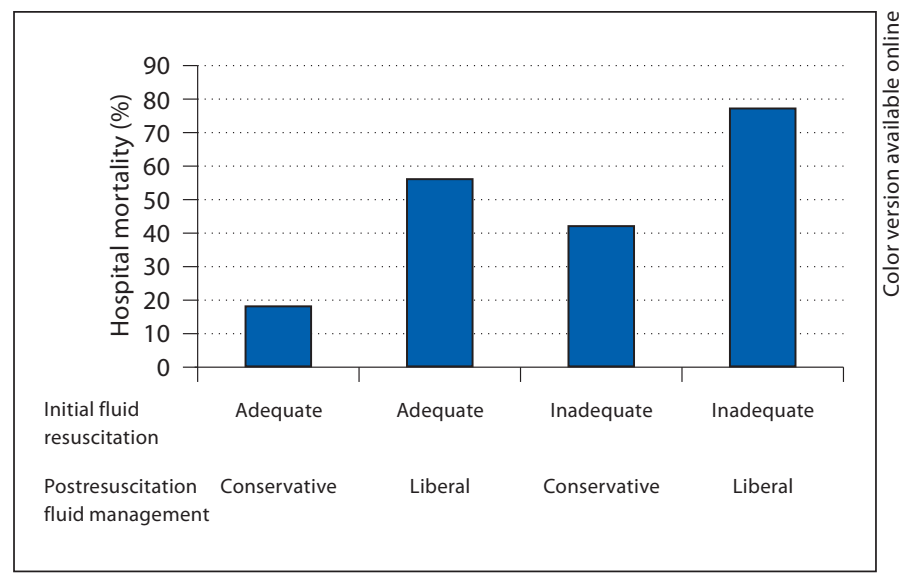

Fig. 1. Effect of adequacy of early fluid resuscitation and late control of fluid overload on mortality in acute lung injury associated with septic shock. $\mathrm{p}<0.001$. Reprinted with permission from Murphy et al. [15].

in Acutely Ill Patients (SOAP) Trial), Vincent et al. [16] compared clinical parameters of 1,177 septic with 1,970 nonseptic patients. Fluid balance was assessed within the first $72 \mathrm{~h}$ of ICU admission, with mean cumulative fluid balance in the septic group during this period being +1.8 vs. +0.5 liters in the nonseptic group. Multivariate regression analysis demonstrated that mean fluid balance during this time period was a significant independent outcome predictor: each 1-liter increase in cumulative fluid balance was associated with a $10 \%$ increase in risk of death.

The above three studies $[14,16,23]$ conducted in septic patients, along with studies performed in other patient populations [17-19], suggest that a positive fluid balance adversely impacts clinical outcomes. Unfortunately, as observational studies, these reports can only establish an association rather than a causal relationship. A recent RCT involving patients with acute lung injury provides substantive evidence of the deleterious effect of fluid overload in critically ill patients. In the Fluid and Catheter Treatment Trial (FACTT) performed by the ARDS Clinical Trial Network investigators [28] with a $2 \times 2$ design, one intervention was the use of a pulmonary artery catheter or a central venous catheter for hemodynamic management. The other intervention was the use of a 'conservative' or 'liberal' fluid management strategy over a 1-week intervention period. The primary endpoint was 60-day mortality while secondary endpoints included ventilator-free days and organ failure-free days. Fluid administration and the use of vasoactive agents and fu- 
rosemide were guided by filling pressures and mean arterial pressure with a complex defined protocol. Approximately 500 patients comprised each of the two fluid management arms. After a week, the average total fluid balance in the conservative group was $-136 \mathrm{ml}$ while in the liberal group it was $+6,992 \mathrm{ml}(\mathrm{p}<0.001)$.

Although survival at 60 days was not significantly different between the two groups, a number of other outcome parameters differed significantly:

- Ventilator-free days and ICU-free days were both significantly higher in the conservative group.

- Cardiovascular failure during the first week occurred at a significantly higher rate in the liberal group than in the conservative group.

- The percentage of patients requiring dialysis at 60 days in the conservative group was lower than in the liberal group ( 10 vs. $14 \%$; $p=0.06$ ).

Although not specifically stated, the most likely dialysis precipitant in the liberal group was fluid overload, given the large net positive fluid balance that occurred in these patients.

\section{Critically Ill Patients with AKI}

As noted above, an association between fluid overload and increased mortality $[10-12,29]$ in critically ill pediatric patients with AKI has been established by several studies. A recent study [13] performed in the adult AKI population not only confirms this association but also elucidates the detrimental impact of AKI on fluid balance.

Payen et al. [13], extracting data from the above referenced SOAP study, analyzed the influence of patient characteristics and fluid balance on the outcome of AKI in critically ill patients. Of the 3,147 patients included in the SOAP study, $36 \%$ had AKI during their ICU stay, with a 60 -day mortality of $36 \%$ compared with $16 \%$ among non-AKI patients. Oliguria (diuresis $<500 \mathrm{ml} /$ day), need for RRT, fluid overload and late initiation of RRT $(>2$ days after ICU admission) were associated with worse outcome. In patients with AKI, mean daily fluid balance was more commonly positive among non-survivors than among survivors.

Among the 1,120 septic patients who developed AKI in the SOAP Study, these investigators reported that mean daily fluid balance was significantly more positive in AKI patients who died compared with those who survived $(0.98 \pm 1.50$ vs. $0.15 \pm 1.06$ liters $/ 24 \mathrm{~h} ; \mathrm{p}<0.001)$. Multivariate regression analysis demonstrated that mean daily fluid balance was a significant and independent mortality predictor: a 1-liter positive fluid balance per
$24 \mathrm{~h}$ was associated with an approximate $20 \%$ increase in mortality risk. Finally, mean daily fluid balance was found to be significantly greater in oliguric patients versus non-oliguric patients $(0.62 \pm 1.33$ vs. $0.27 \pm 1.23$ liters $/ 24 \mathrm{~h} ; \mathrm{p}<0.01)$ and in dialyzed patients versus nondialyzed patients $(0.60 \pm 1.50$ vs. $0.39 \pm 1.21$ liters $/ 24 \mathrm{~h}$; $\mathrm{p}<0.01)$.

A recent study from the PICARD group in the US further establishes an association between fluid overload and mortality among critically ill adult AKI patients [30]. This trial was an observational study in which fluid status was estimated quantitatively in $610 \mathrm{AKI}$ patients. Of these patients, approximately two-thirds (396 patients) required some form of dialysis. Fluid balance was estimated by measuring the sum of all fluid inputs (e.g. intravenous fluids, medications, nutrition) and the sum of all fluid outputs (e.g. urine, stool, nasogastric fluid). On a daily basis, 'fluid accumulation' was calculated as the difference in these sums divided by the body weight at the time of hospital admission. Measurements started 3 days before nephrology consultation and continued until hospital discharge. The study defined 'fluid overload' as a fluid accumulation value of greater than $10 \%$ (i.e. the point at which a patient sustained a fluid-related weight gain of $10 \%$ from baseline).

The patients who met the $10 \%$ fluid overload criterion at the time of AKI diagnosis were more critically ill than patients with lesser degrees of fluid accumulation, as shown by higher APACHE III and SOFA scores, number of organ failures, and percentage of patients with respiratory failure, ventilator dependence, and sepsis or septic shock. It should be noted that the outcomes reported were adjusted for this greater illness severity in the fluid overload group. The following results were reported:

(1) Effect of fluid overload on survival both in patients treated with dialysis and patients treated conservatively: For the patients who received dialysis (either IHD or CRRT), in whom fluid status was assessed at the time of dialysis initiation, survival was significantly higher in those patients without fluid overload (fluid accumulation $<10 \%)$ than in patients with fluid overload. Likewise, in those patients not treated with dialysis, fluid overload at the time of AKI diagnosis was associated with lower survival.

Patients achieving negative fluid balance ('loss') had substantially lower 60-day mortality than patients who had positive fluid balance ('gain'). For each weight change class, the mortality of dialyzed patients was significantly greater than that of nondialyzed patients, consistent with greater illness severity in the former group and suggest- 
ing the existence of a dose-effect relationship. The length of time patients remained in a fluid overloaded state while being treated with dialysis also was a significant determinant of mortality. There was a stepwise increase in mortality as the percentage of dialysis days in a fluidoverloaded state increased from $0 \%$ (mortality, $18 \%$ ) to $100 \%$ (mortality, 62\%). One potential interpretation of these data is that duration of fluid overload is a surrogate for the effectiveness of its correction by a particular dialysis modality.

(2) Superiority of CRRT over IHD in achieving net fluid removal in critically ill AKI patients (fig. 2): Consistent with a greater degree of illness severity, fluid overload at the initiation of therapy was substantially higher in the CRRT group (mean value, approximately 14\%) compared with the IHD group (approximately 11\%). This notwithstanding, net daily fluid removal with CRRT resulted in a progressive decrease in mean fluid accumulation to approximately $8 \%$ after 10 days. Conversely, this mean fluid accumulation progressively increased over time in the IHD group, reaching approximately $18 \%$ after 10 days. These data clearly indicate that IHD is generally incapable of managing fluid balance in critically ill AKI patients who require large fluid volumes (typically 4 liters or more per day) on an obligate basis.

Finally, the study also found that the extent of fluid overload during AKI influences renal recovery. Patients with fluid overload at the time of their highest serum creatinine were significantly less likely to recover renal function than those patients without fluid overload.

The PICARD Study establishes a clear association between fluid overload, increased mortality and decreased renal recovery in critically ill adult AKI patients. The study shows not only that the extent of fluid accumulation influences survival, but also that the duration of fluid overload in dialyzed patients worsens outcomes. The latter finding suggests that the ability of each dialysis modality to achieve net fluid removal may be an indirect determinant of outcome.

The relevance of this finding is also highlighted by the superior effectiveness of CRRT in achieving net fluid removal when compared to IHD, corroborating previous data [31] and further raising questions about the appropriateness of using IHD in critically ill AKI patients. The widespread acceptance of this criterion is shown in the design of the ATN trial [32] where in reflection of current US practice, CRRT was considered almost exclusively the appropriate RRT modality in the hemodynamically unstable critically ill patient with AKI [4]. 


\section{Factors influencing fluid management in acute lung injury}

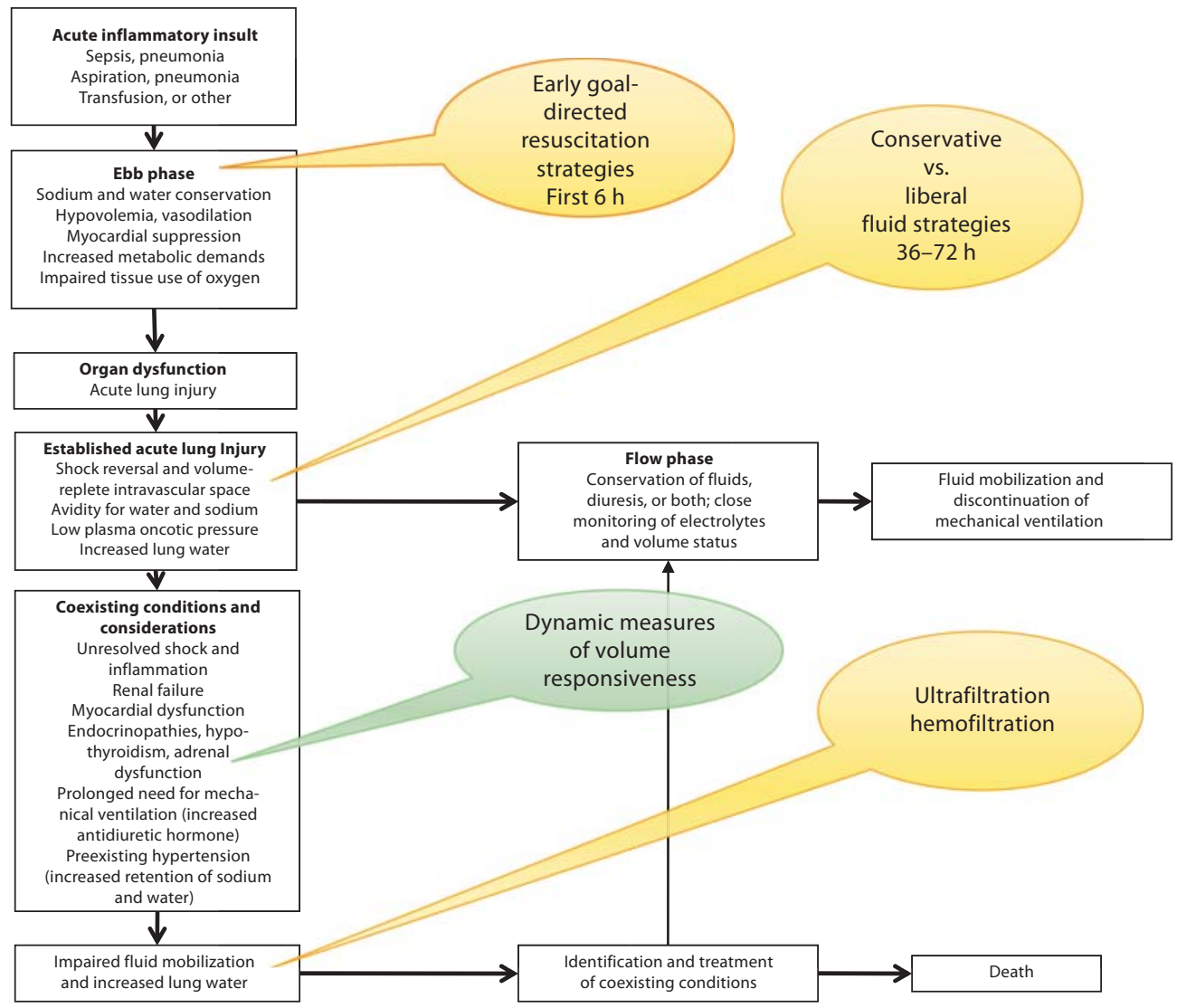

Fig. 3. Proposed time course for fluid balance in acute kidney injury. Modified from Rivers [39].

ated with worse outcomes [13, 28, 33]. Moreover, fluid overload may be the determinant of increased mortality among patients experiencing a decrease in serum creatinine after cardiac surgery [34] and be the reason why in severe AKI, a lower serum creatinine is paradoxically associated with worse prognosis [35].

Finally, several emerging data lines suggest that the approach to overall fluid and hemodynamic management in the setting of septic AKI may need reevaluation $[27,36,37]$. Conventional wisdom and traditional teaching have been that ischemia due to relative hypoperfusion is the predominant mechanism underlying septic AKI. However, recent studies suggest septic and nonseptic AKI have different pathophysiologies and challenge the dictum that the former condition is largely mediated by re- nal hypoperfusion. As such, the rationale for aggressive fluid administration in septic AKI beyond the initial resuscitation phase, as part of routine hemodynamic management and for the express purpose of ameliorating renal ischemia, should be questioned.

Except for the EGDT [23] and FACTT [28] studies, all the studies correlating fluid status and mortality are retrospective. Therefore, it remains to be established whether fluid overload is a causal mechanism or a marker of more severe disease $[22,35]$. The problem is further compounded by our inability to reliably measure fluid status and to predict cardiac output changes when preload is modified [3, 38]. Moreover, resuscitation protocols are not standardized [8] and there is considerable debate as to the optimal approach to fluid and hemodynamic man- 
agement in critical patients with AKI. The recent observations of the PICARD group [30] further give credence to the concept that fluid overload is an independent risk factor for mortality.

These considerations are especially relevant to septic patients, who consistently develop AKI despite fluid expansion [22], and in whom fluid challenges further deteriorate respiratory function. Evaluating the relationship between fluid status and AKI among septic patients, Van Biesen et al. [21] found that septic patients developing AKI show elevated central venous pressure beginning with the first day of sepsis, indicating either cardiodepression or intrarenal hypoperfusion. These patients develop AKI despite fluid loading, suggesting that such challenges should be avoided if they do not lead to improvement in renal function or if oxygenation deteriorates.

These apparently conflicting observations are better understood when seen in the perspective of the timecourse of disease [39] (fig. 3). Thus, while septic patients may initially (within the first $6 \mathrm{~h}$ ) benefit from aggressive volume resuscitation, as disease progresses and system failure develops, a more conservative fluid management strategy is warranted, often including the use of diuretics as long as effective. Given the difficulties in assessing volume status with routine static measures of preload, dynamic techniques for assessment of volume status, such as leg elevation or pulse pressure variation $[38,40,41]$ are most useful at this stage.

If the patient continues to deteriorate and additional organ failures develop, diuretic responsiveness typically ceases. At that time, rather than prolonging ineffective diuretic treatments [8], early institution of ultrafiltration/ renal replacement therapy is usually necessary to avoid the negative impact of fluid overload [4]. Renal replacement techniques such as CRRT offer the best hemodynamic stability in this setting, and facilitate fluid management without hypotension and further renal injury.

As remarked by Rivers [39], fluid may be a friend when appropriately titrated during resuscitation or the ebb phase of acute lung injury but excess fluid becomes an enemy when it is no longer physiologically needed. Conservative fluid management during the established phase of acute lung injury is just as important as titrated liberal administration during the acute phase of the inciting insult. Surgeons have long been aware of this fact, as pointed out by Moore and Shires [42] many decades ago: 'The objective of care is restoration of normal physiology and normal function of organs, with a normal blood volume, functional body water and electrolytes. This can never be accomplished by inundation.'

\section{Conclusions}

In the critically ill patient, persistent fluid overload must be avoided: It does not benefit the patient and there is evidence that it may be harmful.

In the septic patient, early volume expansion seems to be beneficial $[8,39]$. Beyond that threshold, when organ failure develops, fluid overload has been shown to be associated with worse outcomes in multiple disparate studies. One well-designed RCT [28] showed the benefit of a conservative fluid management strategy based on limited fluid intake and use of furosemide in such patients.

Use of diuretics should be only short term as long as it is effective, generally at high doses, while avoiding simultaneous utilization of nephrotoxins such as aminoglycosides. Multiple RCTs have not shown benefit in the use of diuretics, either to prevent AKI or to treat established AKI.

If fluid overload (defined as fluid accumulation $>10 \%$ over baseline) develops and the patient does not respond to diuretics, persistent use of these drugs will only lead to delay in the initiation of dialysis or ultrafiltration and an increased risk for negative patient outcomes. In that setting, early initiation of continuous renal replacement therapies may be preferable [4].

\section{References}

1 Cerda J, Lameire N, Eggers P, et al: Epidemiology of acute kidney injury. Clin J Am Soc Nephrol 2008;3:881-886.

$\checkmark 2$ Cerda J, Bagga A, Kher V, Chakravarthi RM: The contrasting characteristics of acute kidney injury in developed and developing countries. Nat Clin Pract Nephrol 2008;4: 138-153.
3 Kellum JA, Cerda J, Kaplan LJ, Nadim MK, Palevsky PM: Fluids for prevention and management of acute kidney injury. Int J Artif Organs 2008;31:96-110.

-4 Cerda J, Ronco C: Modalities of continuous renal replacement therapy: technical and clinical considerations. Semin Dial 2009;22 114-122.
5 Lameire N, van Biesen W, Hoste E, Vanhold er R: The prevention of acute kidney injury in an in-depth narrative review. Nephrol Dial Transplant 2009;2:1-10.

6 O'Leary MJ, Bihari DJ: Preventing renal failure in the critically ill: there are no magic bullets - just high quality intensive care. BMJ 2001;322:1437-1439. 
7 Mehta RL, Cantarovich F, Shaw A, Hoste E, Murray P: Pharmacologic approaches for volume excess in acute kidney injury (AKI). Int J Artif Organs 2008;31:127-144.

$>8$ Mehta RL: Fluid balance and acute kidney injury: the missing link for predicting adverse outcomes? Nat Clin Pract Nephrol 2009;5:10-11.

$\checkmark 9$ Schetz M: Diuretics in acute renal failure? Contrib Nephrol. Basel, Karger, 2004, vol 144, pp 166-181.

$\checkmark 10$ Foland JA, Fortenberry JD, Warshaw BL, et al: Fluid overload before continuous hemofiltration and survival in critically ill children: a retrospective analysis. Crit Care Med 2004;32:1771-1776.

-11 Gillespie RS, Seidel K, Symons JM: Effect of fluid overload and dose of replacement fluid on survival in hemofiltration. Pediatr Nephrol 2004;19:1394-1399.

-12 Goldstein SL, Currier H, Graf C, Cosio CC, Brewer ED, Sachdeva R: Outcome in children receiving continuous venovenous hemofiltration. Pediatrics 2001;107:1309-1312.

$\checkmark 13$ Payen D, de Pont AC, Sakr Y, et al: A positive fluid balance is associated with a worse outcome in patients with acute renal failure. Crit Care 2008;12:R74.

14 Alsous F, Khamiees M, DeGirolamo A, Amoateng-Adjepong Y, Manthous CA: Negative fluid balance predicts survival in patients with septic shock: a retrospective pilot study. Chest 2000;117:1749-1754.

-15 Murphy CV, Schramm GE, Doherty JA, et al: The importance of fluid management in acute lung injury secondary to septic shock. Chest 2009;136:102-109.

16 Vincent JL, Sakr Y, Sprung CL, et al: Sepsis in European intensive care units: results of the SOAP study. Crit Care Med 2006;34: 344-353.

-17 Brandstrup B, Tonnesen H, Beier-Holgersen $\mathrm{R}$, et al: Effects of intravenous fluid restriction on postoperative complications: comparison of two perioperative fluid regimens: a randomized assessor-blinded multicenter trial. Ann Surg 2003;238:641-648.

- 18 Lowell JA, Schifferdecker C, Driscoll DF, Benotti PN, Bistrian BR: Postoperative fluid overload: not a benign problem. Crit Care Med 1990;18:728-733.
19 Schuller D, Mitchell JP, Calandrino FS, Schuster DP: Fluid balance during pulmonary edema. Is fluid gain a marker or a cause of poor outcome? Chest 1991;100:1068-1075

20 Bagshaw SM, Lapinsky S, Dial S, et al: Acute kidney injury in septic shock: clinical outcomes and impact of duration of hypotension prior to initiation of antimicrobial therapy. Intensive Care Med 2009;35:871-881.

21 Van Biesen W, Yegenaga I, Vanholder R, et al: Relationship between fluid status and its management on acute renal failure (ARF) in intensive care unit (ICU) patients with sepsis: a prospective analysis. J Nephrol 2005; 18 : 54-60.

22 Bagshaw SM, Brophy PD, Cruz D, Ronco C: Fluid balance as a biomarker: impact of fluid overload on outcome in critically ill patients with acute kidney injury. Crit Care 2008;12: 169.

23 Rivers E, Nguyen B, Havstad S, et al: Early goal-directed therapy in the treatment of severe sepsis and septic shock. N Engl J Med 2001;345:1368-1377.

24 Dellinger RP, Levy MM, Carlet JM, et al: Surviving Sepsis Campaign: international guidelines for management of severe sepsis and septic shock: 2008. Crit Care Med 2008; 36:296-327.

25 Hicks P, Cooper DJ, Webb S, et al: The Surviving Sepsis Campaign: international guidelines for management of severe sepsis and septic shock: 2008. An assessment by the Australian and New Zealand intensive care society. Anaesth Intens Care 2008;36:149151.

26 Bagshaw SM, Uchino S, Bellomo R, et al: Septic acute kidney injury in critically ill patients: clinical characteristics and outcomes. Clin J Am Soc Nephrol 2007;2:431-439.

$\checkmark 27$ Wan L, Bagshaw SM, Langenberg C, Saotome T, May C, Bellomo R: Pathophysiology of septic acute kidney injury: what do we re ally know? Crit Care Med 2008;36:S198S203.

28 Wiedemann HP, et al: National Heart Lung, Blood Institute Acute Respiratory Distress Syndrome Clinical Trials. Comparison of two fluid-management strategies in acute lung injury. N Engl J Med 2006;354:25642575

-29 Goldstein SL, Somers MJ, Baum MA, et al: Pediatric patients with multi-organ dysfunction syndrome receiving continuous renal replacement therapy. Kidney Int 2005;67: 653-658.
30 Bouchard J, Soroko SB, Chertow GM, et al: Fluid accumulation, survival and recovery of kidney function in critically ill patients with acute kidney injury. Kidney Int 2009;76: 422-427.

31 Augustine JJ, Sandy D, Seifert TH, Paganini EP: A randomized controlled trial comparing intermittent with continuous dialysis in patients with ARF. Am J Kidney Dis 2004;44: 1000-1007.

32 Network VNARFT, Palevsky PM, Zhang JH, et al: Intensity of renal support in critically ill patients with acute kidney injury. N Engl J Med 2008;359:7-20.

>33 Bagshaw SM, Bellomo R, Kellum JA: Oliguria, volume overload, and loop diuretics. Crit Care Med 2008;36:S172-S178.

-34 Lassnigg A, Schmidlin D, Mouhieddine M, et al: Minimal changes of serum creatinine predict prognosis in patients after cardiothoracic surgery: a prospective cohort study. J Am Soc Nephrol 2004;15:1597-1605.

>35 Cerda J, Cerda M, Kilcullen P, Prendergast J: In severe acute kidney injury, a higher serum creatinine is paradoxically associated with better patient survival. Nephrol Dial Transplant 2007;22:2781-2784.

36 Licari E, Calzavacca P, Ronco C, Bellomo R: Fluid resuscitation and the septic kidney: the evidence. Contrib Nephrol 2007;156:167177.

37 Langenberg C, Wan L, Egi M, May CN, Bellomo R: Renal blood flow and function during recovery from experimental septic acute kidney injury. Intens Care Med 2007;33: 1614-1618.

38 Pinsky MR, Brophy P, Padilla J, Paganini E, Pannu N: Fluid and volume monitoring. Int J Artif Organs 2008;31:111-126.

39 Rivers EP: Fluid-management strategies in acute lung injury - liberal, conservative, or both? N Engl J Med 2006;354:2598-2600.

40 Vincent JL, Pinsky MR, Sprung CL, et al: The pulmonary artery catheter: in medio virtus. Crit Care Med 2008;36:3093-3096.

41 Pinsky MR: Hemodynamic evaluation and monitoring in the ICU. Chest 2007;132: 2020-2029.

42 Moore FD, Shires GT: Moderation. Ann Surg 1967;166:300-3001. 